\title{
Investigation of wave generation and propagation during ultrasound-excited thermography testing and its effects on defect detection
}

\author{
by S. Kishimoto*, M. Ishikawa*, H. Nishino*, R. Fukui ${ }^{\star \star}$, Y. Habuka** and Y. Nishitani** \\ * Tokushima University, 2-1 Minamijousanjimacho, 770-8506, Tokushima, Japan \\ ** KJTD Co., Ltd., 45th floor Sunshine60, 3-1-1 Higashiikebukuro, Toshima-ku,Tokyo,170-6045, Japan
}

\begin{abstract}
In this study, wave propagation during the ultrasound-excited thermography is examined mainly from the viewpoint of the elastic wave propagation study. When ultrasonic waves are input in a test object, various kinds of waves are excited, and standing waves are often generated depending on the wavelength of the input ultrasound and the shape of the tested object. We investigated the generation process of the standing waves and its influence on defects detection through experiments and numerical calculations.
\end{abstract}

\section{Introduction}

Ultrasound-excited thermography is a nondestructive inspection method used to detect defects on and near the surface of test objects. This method can inspect a large area in a short time, and is known as of an effective method for detection of closed defects such as cracks [1-5]. In this method, defects are detected as frictional heats generated by the vibration of the propagated ultrasounds [6,7]. When ultrasonic waves are input in a test object, various kinds of waves (such as longitudinal waves, traverse waves, and plate waves) are excited, and, depending on the wavelength of the ultrasonic waves and the shape of the tested object, standing waves are often generated. Thus, to understand the wave propagation and its effect on defect detection is important for proper understanding of the inspection results. In this study, we focused on the generation process of standing waves, and investigated its effect on observed temperature images based on the consideration of the thermoelastic effect. Moreover, the influence of the standing waves on defect detection was studied through experiments and numerical calculations.

\section{Effect of standing waves on observed temperature images}

\subsection{Experiments} was performed.

In order to investigate the effect of standing waves in observed temperature images, an experiment for a specimen

\subsubsection{Experimental setup}

A picture and a schematic diagram of the experimental setup are shown in figure 1. In the experiments, an acrylic plate $(150 \times 100 \times 3 \mathrm{~mm})$ without any defect was used as a specimen. Ultrasonic waves were excited in the specimen using an ultrasonic welder (W3040, Nippon Future Co., Ltd.) through an ultrasonic horn with a diameter of $15 \mathrm{~mm}$. The output power and the nominal excitation frequency of the ultrasonic welder were $1200 \mathrm{~W}$ and $20 \mathrm{kHz}$, respectively. The ultrasonic horn was directly pressed to the specimen, and the specimen was fixed between the horn and a rubber material $(20 \times 20 \times 10 \mathrm{~mm})$ placed under the specimen. The ultrasound was excited for $0.3 \mathrm{~s}$, and the surface temperature was observed for $1 \mathrm{~s}$ with an infrared camera (SC7500, FLIR Systems Co.). The frame rate of the infrared camera was 100 $\mathrm{Hz}$. Note that the surface of the specimens was painted with a matte black spray to reduce disturbance from the surrounding during observation and to improve temperature measurement accuracy.

\subsubsection{Experimental results}

Figure 2(a) shows an obtained temperature image. Although there are no defects in the specimen, periodic temperature rises are observed through the specimen surface. This temperature change is caused by the standing wave generated in the specimen; heat should be generated in the areas corresponding to antinodes of the standing wave, and temperature distributions caused by the heat generation is observed. The temperature-time relation observed at the point indicated in figure 2(a) is shown in figure 2(b). The temperature increased more than $1^{\circ} \mathrm{C}$. If there is no consideration for the effect of the standing wave, such temperature rise could be misunderstood as the temperature change caused by defects. 
(a)

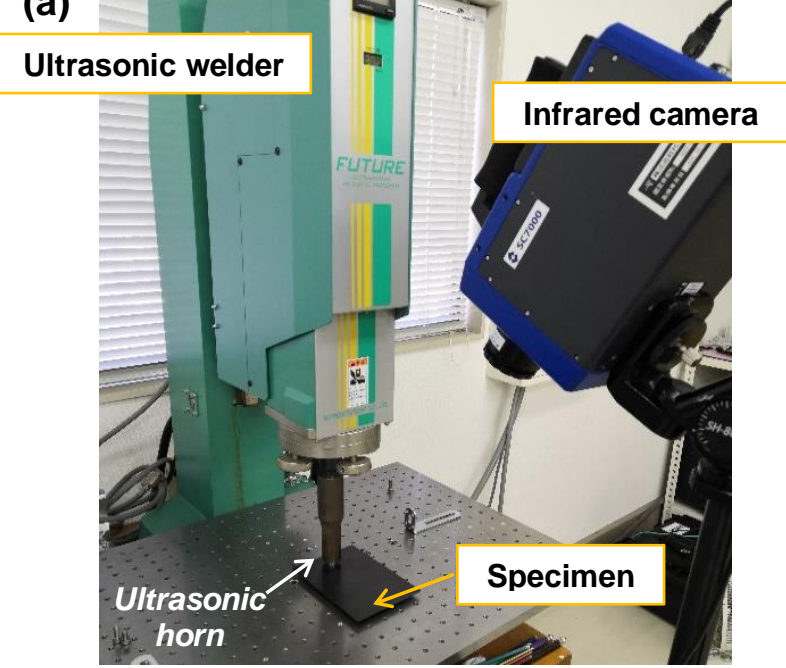

(b)

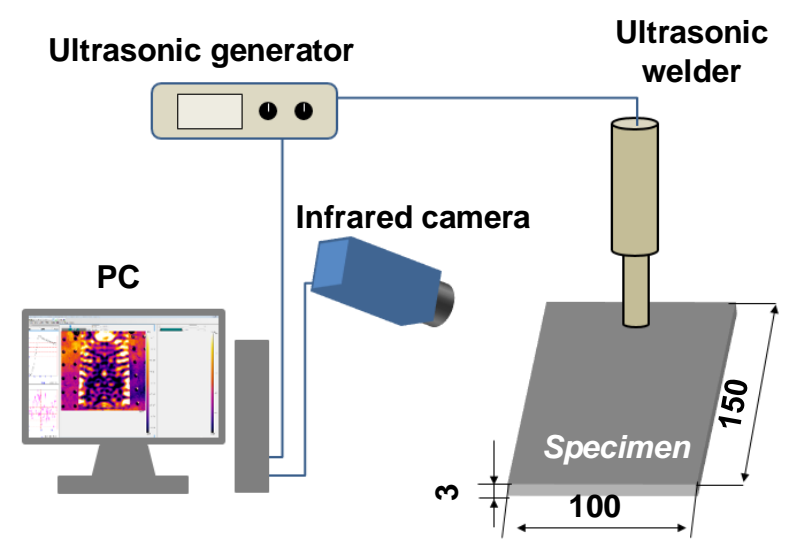

Fig. 1. (a) a picture and (b) a schematic of the experimental setup.

(a)

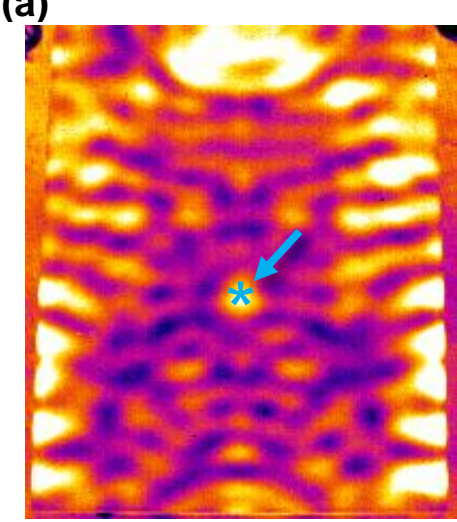

$25.16\left[^{\circ} \mathrm{C}\right]$

25.15

24.65

24.15

23.65 (b)

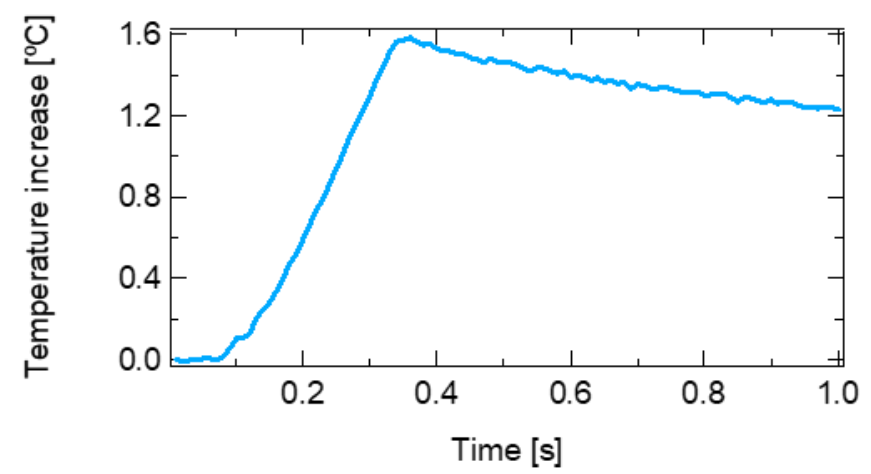

Fig. 2. (a) Experimentally obtained temperature image at $0.3 \mathrm{~s}$, and (b) temperature increase at the point indicated in Fig. 2(a) as a function of time.

\subsection{Numerical studies}

The temperature distribution observed in the experiment was verified using numerical calculations.

\subsubsection{Numerical setup}

Calculations were performed using a commercial finite element method software (ANSYS 18.0). The calculation model simulated the experimental setup described in the previous section (figure 1(a)). To simulate the ultrasonic excitation, a cyclic forced displacement was applied to the area where the ultrasonic horn was placed in the experiment. The cyclic frequency was $20 \mathrm{kHz}$, and the excitation time was $30 \mathrm{~ms}$. The material properties used in the calculations (simulating the acrylic resin) are shown in Table 1 . In the calculations, temperature change caused by the thermoelastic effect was considered to calculate the temperature distribution caused by the cyclic deformation.

Table 1. Material properties used in calculations.

\begin{tabular}{|l|c|}
\hline Density $\left[\mathrm{kg} / \mathrm{m}^{3}\right]$ & 1190 \\
\hline Elastic Modulus [GPa] & 3.0 \\
\hline Poisson's ratio [-] & 0.35 \\
\hline Thermal conductivity [W/m* $\mathrm{K}]$ & 0.21 \\
\hline Specific heat $\left[\mathrm{J} / \mathrm{kg}^{*} \mathrm{~K}\right]$ & 1400 \\
\hline CTE $[\mathrm{ppm} / \mathrm{K}]$ & 70 \\
\hline
\end{tabular}




\subsubsection{Numerical results}

The calculated temperature distributions at two different time is shown in figure 3 . In both images, periodic temperature pattern is observed on the entire surface of the specimen. Figure 4 shows the surface stress and temperature changes over time at the point indicated in figure 3(a). It is found from figure 4(a) that the amplitude of the surface stress is approximately constant after $5 \mathrm{~ms}$, this means that the vibration reaches a steady state and standing wave is generated at $5 \mathrm{~ms}$. Also in the temperature data (figure 4(b), cyclic temperature change is observed, and its amplitude becomes constant at around $5 \mathrm{~ms}$. This temperature change is caused by the cyclic stress variation shown in figure 4(a) and the thermoelastic effect. It is worth noting in figure 4(b) that, in addition to the cyclic change, the base line of the temperature gradually increases with time. This should be because of the accumulation and propagation of the generated heat. Figure 5 shows the temperature distribution obtained by subtracting the temperature values in figure 3(a) from those in figure 3(b) (i.e., figure 5 shows the distribution of the increment of the base line for approximately $20 \mathrm{~ms}$ after the standing wave is generated). By comparing with figure 2(a), it is found that figure 5 is similar to the experimentally obtained temperature. These results imply, although it is difficult to observed the instantaneous temperature variation caused by the thermoelastic effect (such as observed in figure 3), continuous change of the base line of the temperature variation is observed in the experiment

(a)

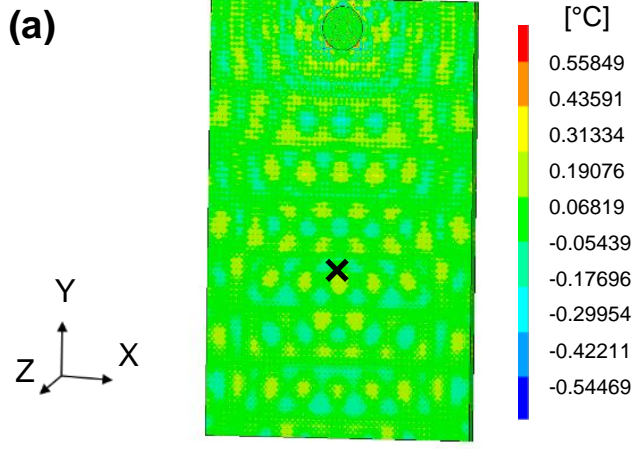

(b)

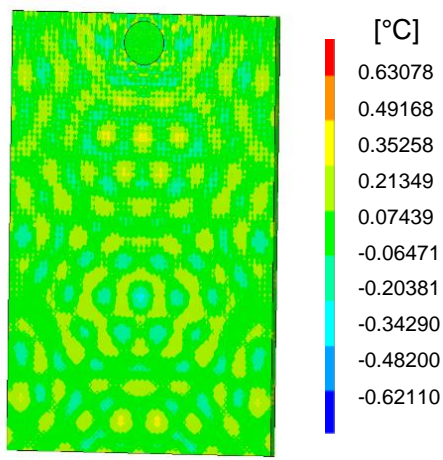

Fig. 3. Numerically obtained temperature distribution at (a) $11.50 \mathrm{~ms}$ (b) $31.99 \mathrm{~ms}$ after the start of excitation.

(a)

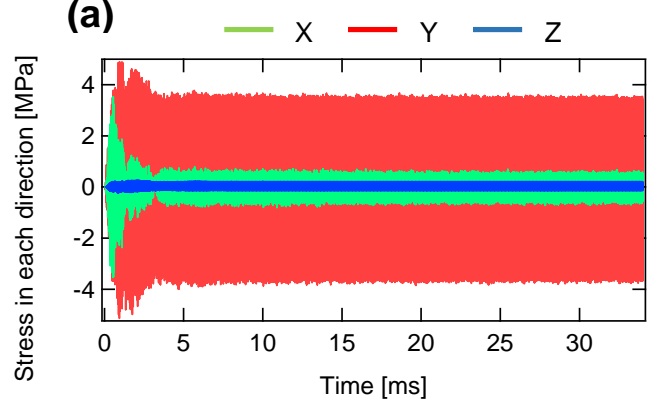

(b)

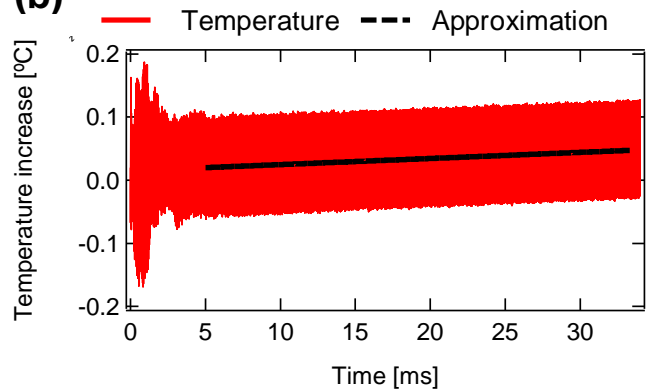

Fig. 4. Numerically obtained values at the point indicated in Fig. 3(a):

(a) stress in each direction, (b) temperature change as a function of time.

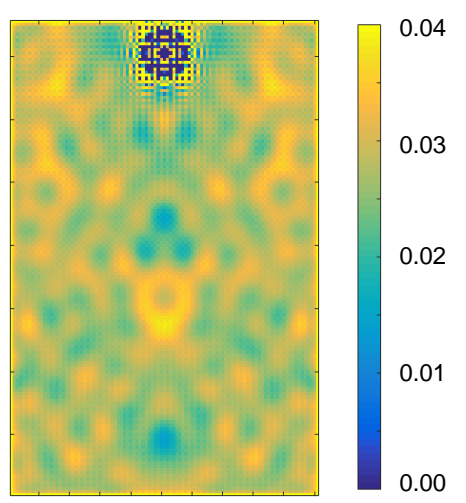

Fig. 5. Numerically obtained temperature distribution obtained by subtracting the temperature values in figure $3(a)$ from those in figure 3(b). 


\section{Effects of standing waves on defect detection}

\subsection{Numerical study on the influence of defect length on generation of standing waves}

When a test object has a defect, the distribution of the standing wave could be varied, and the observed temperature distribution is also influenced. The standing wave distribution could be varied depending on the size of the defect. Therefore, the influence of the defect size on the generated standing wave was investigated through numerical harmonic response analysis.

Figure 6 shows a schematic of the numerical model. The model was similar to that described in the section 2.2 but had a thin slit as a defect. The slit width was $1 \mathrm{~mm}$, and its length was varied as 10, 20, 30, 40, and $50 \mathrm{~mm}$. The ultrasonic excitation was simulated by applying a cyclic forced displacement with a frequency of $28 \mathrm{kHz}$. The material properties were the same as presented in Table 1. In addition, a model without any defect was calculated for comparison. From the calculation, relationship between the surface displacement distribution, which reflects the temperature distribution caused by the standing wave, and the defect length was investigated.

Figure 7 shows the calculated surface displacement distributions for the models. The displacement distributions of the models with the defect (figure $7(\mathrm{~b})-(\mathrm{f})$ ) show similar tendency to that without the defect (figure 7(a)) regardless of the defect length. This should be because the width and length of the defect is smaller than the wavelength of the input ultrasound (approximately $100 \mathrm{~mm}$ ). However, the displacement distribution varies in detail with the change of defect length; the influence of the defect is relatively noticeable when the defect length is $50 \mathrm{~mm}$ (figure 7(f)).

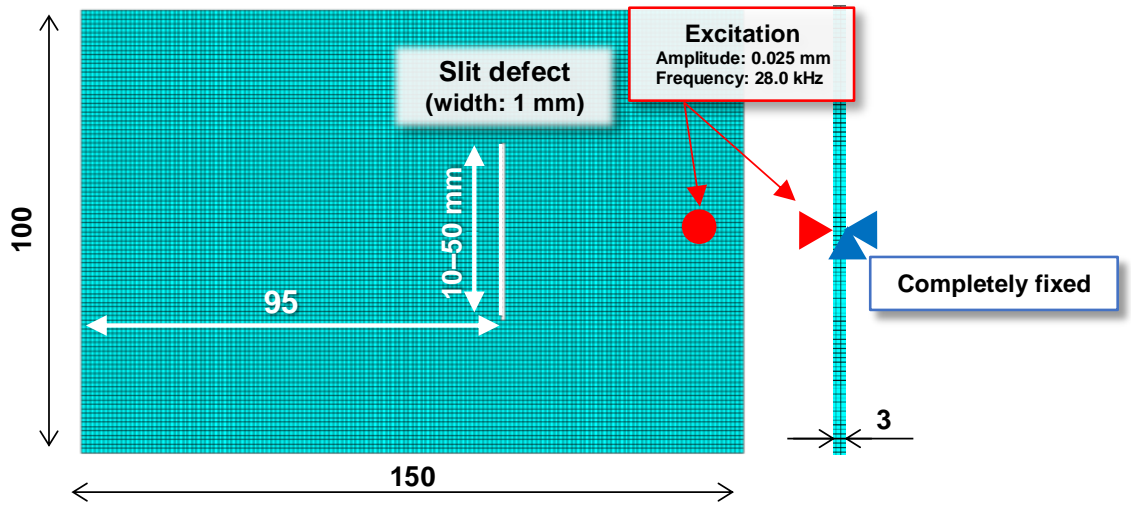

Fig. 6. Schematic diagram of the calculation model with a slit defect.

\subsection{Experiment for a specimen with a defect}

The influence of a defect on the generation of standing wave was experimentally verified. Figure 8 shows a picture of the specimen with a crack defect. The specimen was an acrylic plate $(150 \times 100 \times 3 \mathrm{~mm})$, and had a defect introduced by a high speed impact of a small plastic ball (a $\varphi 6 \mathrm{~mm}$ ball bullet). The defect was a closed crack and the maximum length was approximately $20 \mathrm{~mm}$. Ultrasound was excited in the specimen using a handy-type ultrasonic welder (W-2005-28, Nippon Future Co., Ltd.). The output power and nominal excitation frequency of the welder were $180 \mathrm{~W}$ and $28 \mathrm{kHz}$, respectively, and the ultrasound was input through an ultrasonic horn with a diameter of $6 \mathrm{~mm}$. The ultrasonic horn was directly pressed to the specimen, and the specimen was fixed between the horn and a rubber material $(10 \times 10 \times 10 \mathrm{~mm})$ placed under the specimen. The ultrasound was excited for $5 \mathrm{~s}$, and the surface temperature during and after the excitation was observed using an infrared camera (A315, FLIR Systems Co.) at a frame rate of $60 \mathrm{~Hz}$. In addition to the specimen with a defect, an experiment for a specimen without any defect was performed in the same condition for comparison.

Figure 9 shows the temperature distributions of the specimens with and without a defect. The periodic temperature distribution caused by the standing wave is observed in both images, and in addition to the effect of the standing wave, a local temperature rise is observed at the defective area in figure 9(b). As expected from the numerical calculations, the generated standing wave is similar regardless of whether the specimen has a defect or not because the defect length is less than the ultrasonic wavelength; this should be the reason for that the observed periodic temperature pattern is similar. Therefore, in this condition, the defective area is easily detected by comparing the thermal images of the specimens with and without defect. 
(a)

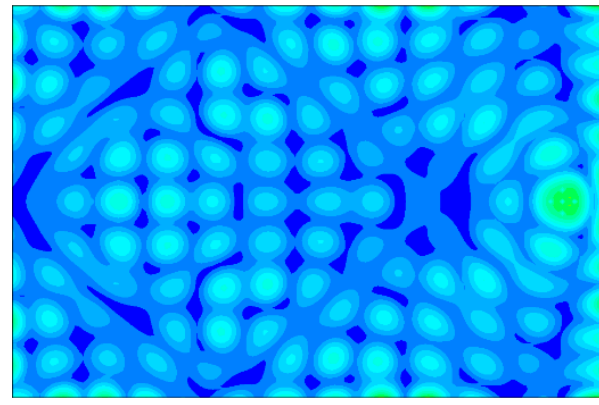

(c)

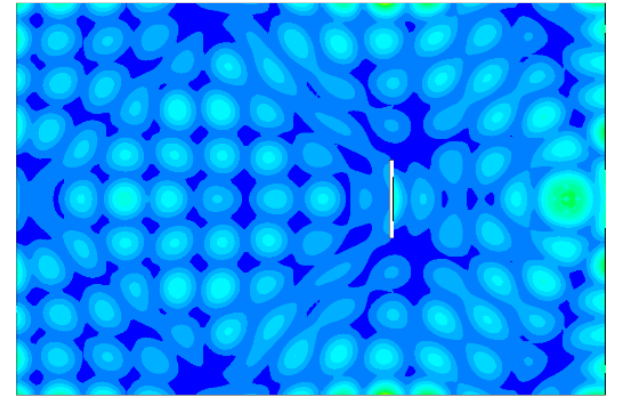

(e)

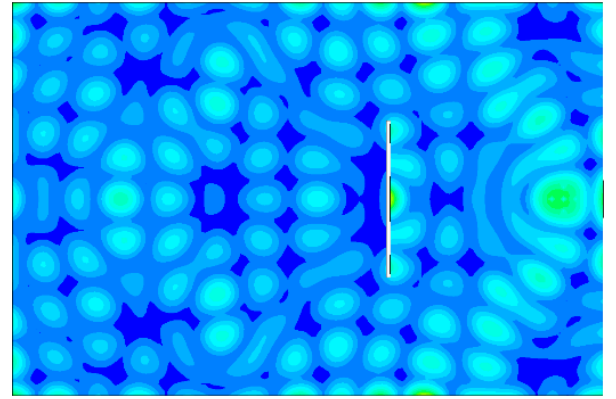

(b)

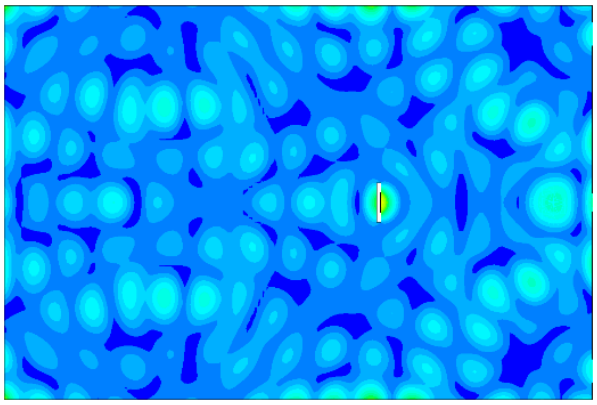

(d)

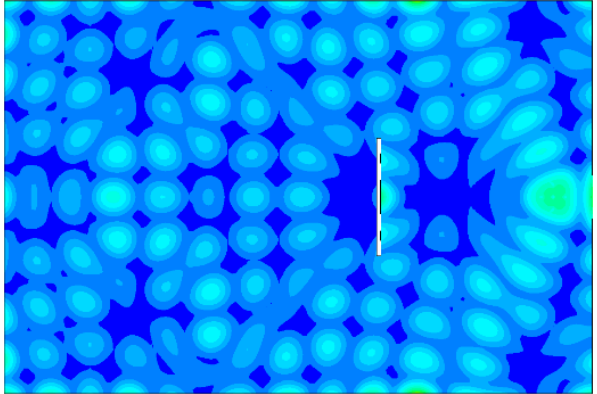

(f)

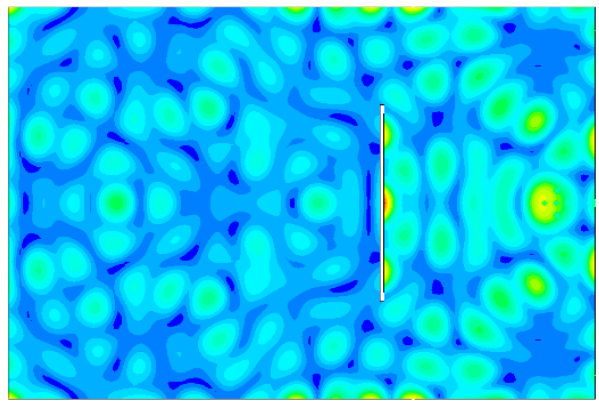

56.2

$84.4 \mu \mathrm{m}$

Fig. 7. Numerically obtained displacement distribution of the model (a) without defect, (b) with a 10-mm-slit (c) with a 20$\mathrm{mm}$-slit, (d) with a 30-mm-slit (e) with a 40-mm-slit, and (f) with a 50-mm-slit.

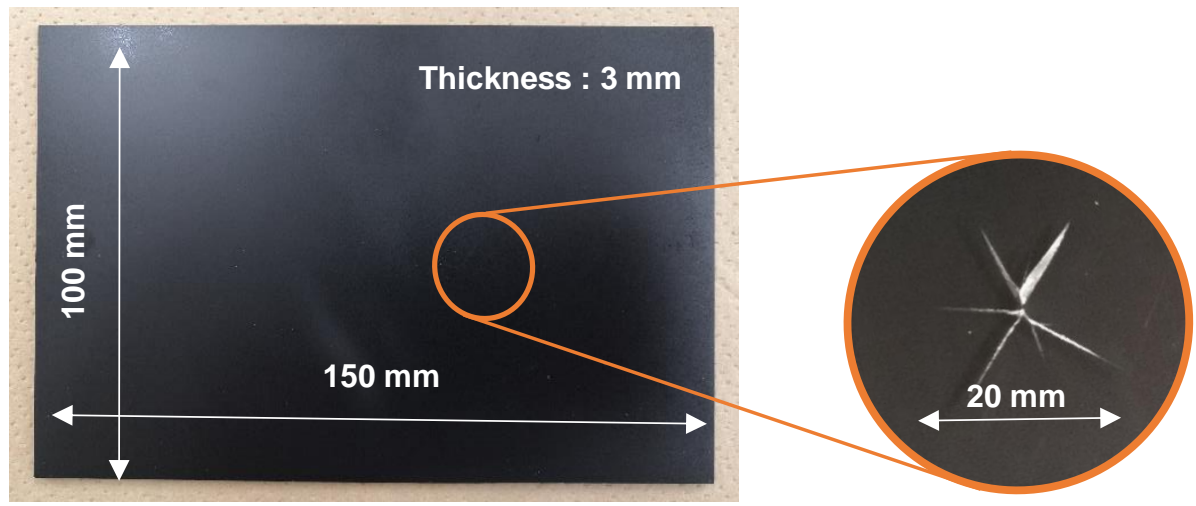

Fig. 8. Acrylic specimen with an arterial defect. 

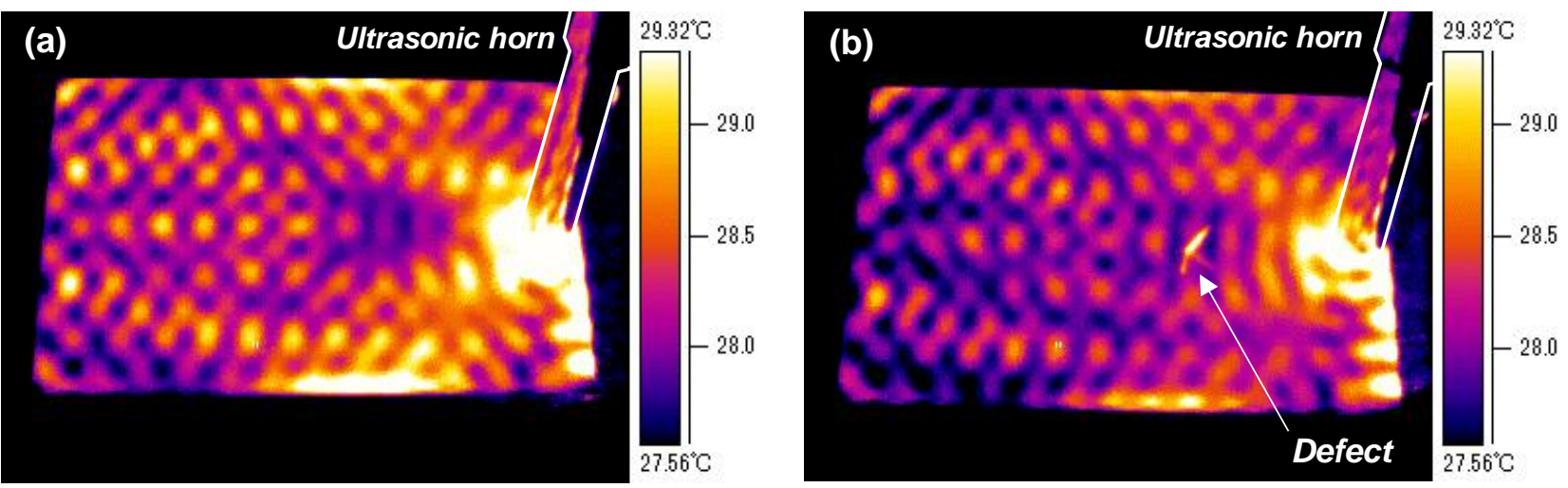

Fig. 9. Experimentally obtained temperature distribution for specimen (a) without defect (b) with an artificial defect.

\section{Conclusions}

In this paper, experimental and numerical studies to investigate the influence of the standing wave on the defect detection using ultrasound-excited thermography were presented. When a standing wave is generated, a periodic temperature pattern is observed, caused by the thermoelastic effect. Because these temperate pattern is observed also in the non-defective region, prior knowledge on the standing waves is important to prevent misunderstandings of the inspection results. Our results showed that the generation of the standing waves is influenced little by defects when the defect size is enough less than the wavelength of the input ultrasonic wave. Conversely, when the defect is larger, the temperature pattern caused by the standing waves is varied and detecting defects in the obtained temperature data becomes more difficult. Therefore, it is necessary to study an effective method of dealing with the effect of standing waves as a future work.

\section{REFERENCES}

[1] Favro, L.D., Thomas, R.L., Han, X., Ouyang, Z., Newaz, G., Gentile, D., Sonic infrared imaging of fatigue cracks, International journal of fatigue, Vol. 23, pp. 471-476, 2001.

[2] Favro L.D., Han X., Ouyang Z., Sun G., and Thomas R.L., Sonic IR Imaging of cracks and delaminations, In Analytical Sciences/Supplements Proceedings of 11th International Conference of Photoacoustic and Photothermal Phenomena (pp. s451-s453), 2002.

[3] Han, X., Favro, L.D., Thomas, R.L., Sonic IR imaging and vibration pattern studies of cracks in an engine disk, In AIP conference proceedings (Vol. 657, No. 1, pp. 513-516), 2003.

[4] Morbidini, M., Cawley, P., The detectability of cracks using sonic IR, Journal of Applied Physics, Vol. 105. No. 9, 093530, 2009.

[5] Guo, X., Vavilov, V., Crack detection in aluminum parts by using ultrasound-excited infrared thermography, Infrared Physics \& Technology, Vol. 61, pp. 149-156, 2013.

[6] Renshaw, J., Chen, J.C., Holland, S.D., Thompson, R.B., The sources of heat generation in vibrothermography, NDT \& E International, Vol. 44, no. 8, pp. 736-739, 2011.

[7] Rizi, A.S., Hedayatrasa, S., Maldague, X., Vukhanh, T., FEM modeling of ultrasonic vibrothermography of a damaged plate and qualitative study of heating mechanisms, Infrared Physics \& Technology, Vol. 61, pp. 101110, 2013. 